Alchemilla subsericea, Lonicera nigra, Viola palustris. - Aus Oberösterreich: Cardamine Hayneana, Pulmonaria mollis.

Obige Pflanzen können nach beliebiger Auswahl im Tausche oder käuflich die Centurie zu 6 fl. (12 R. Mark) abgegeben werden.

\title{
Einladung zur Pränumeration
}

\author{
auf den XXXIII. Jahrgang (1883)
}

der

Oesterreichischen

\section{Botanischen Zeitschrift.}

(0esterr. botan. Wochenblatt.)

Auf die „Oesterreichische botanische Zeitschrift", welche von dem hohen k. k. österreichischen und dem hohen k. ungarischen Ministerium für Cultus und Unterricht den Mittelschulen empfohlen wurde, pränumerirt man mit 8 fl. österr. W. (16 R. Mark) auf den ganzen Jahrgang oder mit 4 fl. österr. W. (8 R. Mark) auf einen Semester und zwar auf Exemplare, die frei durch die Post bezogen werden sollen, nur bei der Redaction: Wien, IV. Mühlgasse Nr. 1.

Alle Buchhandlungen des In- und Auslandes nehmen ebenfalls Pränumerationen an. Die Versendung an die Buchhandlungen hat die Verlagshandlung C. Gerold's Sohn in Wien übernommen.

Von den bereits erschienenen Jahrgängen kömmen noch vollständige Exemplare gegen nachfolgende Preise bezogen werden: 2. und 3. Jahrgang zu 1 fl. (2 R. Mark) - 9. bis 22. Jahrgang zu 2 fl. (4 R. Mark) - 23. bis 31. Jahrgang zu 5 fl. (10 R. Mark) 32. Jahrgang 8 fl. (16 R. Mark). Bei Abnahme sämmtlicher Jahrgänge yon der Redaction, 20 Procent Nachlass.

Einzelne Hefte können nur vom laufenden und letztvergangenen Jahrgange abgegeben werden.

Von den bisher erschienenen 27 Porträts der ,Gallerie österreichischer Botaniker" können einzelne Exemplare und zwar in Octav à $50 \mathrm{kr}$. (1 R. Mark) und in Folio auf chin. Papier à 1 fl. (2 R. Mark) abgegeben werden.

Skofitz.

(IV. Mühlgasse Nr. 1.) 